\title{
Long-term effects of gestational diabetes on offspring health are more pronounced in skeletal growth than body composition and glucose tolerance
}

\author{
Jinping Zhao and Hope A. Weiler* \\ School of Dietetics and Human Nutrition, McGill University, Ste-Anne-de-Bellevue, QC, Canada H9X 3V9 \\ (Received 19 January 2010 - Revised 28 May 2010 - Accepted 1 June 2010 - First published online 9 July 2010)
}

Infants of diabetic mothers may have low arachidonic acid (AA) and develop obesity and insulin resistance in adulthood. The present study tested the effect of maternal diabetes and AA supplementation on offspring body composition, bone mass and glucose tolerance from 4 to 12 weeks. Rat dams were randomised into six groups using a $3 \times 2$ design. The rat dams were treated using the following treatments: saline-placebo, streptozotocin-induced diabetes (STZ) with glucose controlled at $<13 \mathrm{mmol} / \mathrm{l}$ (STZ/GC) or poorly controlled at $13-20 \mathrm{mmol} / \mathrm{l}$ (STZ/PC) using insulin, and fed either a control or an AA ( $0.5 \%$ of fat) diet throughout reproduction. Weaned offspring were fed regular chow. Measurements included offspring body composition, bone and oral glucose tolerance testing (OGTT) plus liver fatty acids of dam and offspring. Comparable to salineplacebo offspring, the STZ/GC offspring had greater $(P<0.03)$ whole body and regional bone area than STZ/PC offspring. Maternal glucose negatively correlated $(P<0.05)$ with offspring whole body bone area and mineral content at 4 weeks in all offspring, and with tibia area in males at 12 weeks. Maternal liver DHA negatively $(P<0.03)$ correlated with femur and tibia mineral content and tibia mineral density of female offspring at 12 weeks. Offspring from AA-supplemented dams had higher $(P=0.004)$ liver AA at 4 weeks. Liver AA at 4 weeks positively $(P=0.05)$ correlated with lumbar spine mineral density in males. OGTT was not affected by maternal treatment or diet. These results suggest that maternal glucose control has long-term consequences to bone health of adult offspring. Skeletal growth appears more sensitive to maternal hyperglycaemia than glucose tolerance.

Offspring of diabetic mothers: Maternal arachidonic acid supplementation: Glucose tolerance test: Bone area

The prevalence of diabetes mellitus (DM) in pregnancy is rising in part due to the sharp increase in obesity and type $2 \mathrm{DM}^{(1)}$. Fetal exposure to hyperglycaemia during pregnancy can have profound and long-lasting consequences to the offspring ${ }^{(2)}$. In the Northwestern University Diabetes in Pregnancy follow-up study, $40 \%$ of offspring of diabetic mothers at the age of 16 years had impaired glucose tolerance, defined as blood glucose $7.8-11.0 \mathrm{mmol} / \mathrm{l} 2 \mathrm{~h}$ after receiving a $75 \mathrm{~g}$ glucose $\operatorname{load}^{(3)}$. Furthermore, the occurrence of impaired glucose tolerance was associated with higher insulin in amniotic fluid $^{(4)}$. Based on the SEARCH Case-Control study, $30.4 \%$ of youth with type $2 \mathrm{DM}$ were exposed to maternal diabetes in utero compared with $6.3 \%$ of non-diabetic control youth ${ }^{(5)}$.

Whether altered maternal-fetal nutrient transfer or fetal metabolism explains the propensity for offspring of mothers with DM to develop obesity, impaired glucose tolerance and DM is not clear. In cell culture, arachidonic acid (AA) is fundamental to functional integrity of the pancreatic $\beta$-cell ${ }^{(6)}$, while glucose-stimulated insulin release is dependent on the plasma membrane release of $\mathrm{AA}^{(7)}$. Furthermore, insulin sensitivity is positively associated with higher AA status in human subjects ${ }^{(8)}$. In rats, dietary AA supplementation lowers fasting blood glucose and insulin concentrations ${ }^{(9)}$, prevents high-fat diet-induced insulin resistance ${ }^{(10)}$ and enhances glucose disposal ${ }^{(11)}$. However, women with DM have lower AA in erythrocytes, particularly if obese ${ }^{(12)}$, and newborn infants of women with DM also have reduced AA in cord blood $^{(13-18)}$. Lower neonatal status is likely a reflection of lower maternal AA status, placental sequestration of AA and increased fetal utilisation ${ }^{(13)}$. Several studies have explored the possibility of maternal dietary intervention in modifying the susceptibility of offspring to adult diseases ${ }^{(19-21)}$. The differences in body weight and fasting insulin levels in adult rat offspring relate to the $n-6: n-3$ fatty acid ratio in the maternal diet during the perinatal period ${ }^{(21)}$. Similar effects have also been observed in diabetic pregnant rats and their adult offspring ${ }^{(22)}$. We thus hypothesised that maternal supplementation with AA during pregnancy and lactation would prevent subsequent development of impaired glucose tolerance in the offspring at young adult age.

New evidence suggests that there are linkages between glucose metabolism and bone metabolism ${ }^{(23)}$. Since newborn infants of diabetic mothers have compromised bone growth and mineralisation ${ }^{(24-26)}$, as well as reduced AA status ${ }^{(13-18)}$, we hypothesised that maternal AA supplementation may also be beneficial to offspring body composition and bone health.

Abbreviations: AA, arachidonic acid; AUC, area under the curve; BMC, bone mineral content; BMD, bone mineral density; DM, diabetes mellitus; DXA, dualenergy X-ray absorptiometry; OGTT, oral glucose tolerance test; PN, postnatal; STZ, streptozotocin-induced diabetes; STZ/GC, STZ with good controlled; STZ/PC, STZ with poor controlled.

* Corresponding author: Dr H. Weiler, fax +1 514398 7739, email hope.weiler@mcgill.ca 
AA is a precursor to $\mathrm{PGE}_{2}$ that has a biphasic effect on bone by enhancing bone formation at lower concentrations in animals while stimulating resorption at higher concentrations ${ }^{(27)}$. Alteration in the precursor pool affects $\mathrm{PGE}_{2}$ metabolism, while modification of dietary fatty acids leads to parallel alterations in bone fatty acids ${ }^{(28)}$ and $\mathrm{PGE}_{2}$ metabolism $^{(29,30)}$. In a recent study, serum AA was positively associated with whole body bone mass in 8-year-old children ${ }^{(31)}$. Dietary $\gamma$-linoleic acid (a precursor to AA) supplementation enhanced bone mass in young male rats ${ }^{(32)}$, and was effective against diabetes-induced fetal bone development defects ${ }^{(33)}$. Similarly $^{(21)}$, variations in fatty acid composition in the maternal diet during the perinatal period caused changes in bone growth in adult rat offspring ${ }^{(34,35)}$. Thus, in the present follow-up study, we sought to define the effect of maternal diabetes and AA supplementation on offspring body composition including bone mass and glucose tolerance up to 12 weeks of age.

\section{Materials and methods}

\section{Dam streptozotocin-induced diabetes treatment and dietary} arachidonic acid supplementation

The study design, time course and diet composition (Table 1) have been published in detail ${ }^{(36)}$. Briefly, 9-week-old female Sprague-Dawley rats (University of Manitoba breeding colony) were randomised into six groups using a $3 \times 2$ design (five animals/group) and permitted a 1-week adaptation period while being fed the control diet. Diabetes was induced on week 1 using streptozotocin (STZ) $(60 \mathrm{mg} / \mathrm{kg}$ intraperitoneally; Sigma-Aldrich, St Louis, MO, USA); control received an equivalent amount of saline denoted as saline-placebo. Diabetes was confirmed by blood glucose $>13 \mathrm{mmol} / \mathrm{l}$ using a glucometer (One Touch ${ }^{\circledR}$ Ultra ${ }^{\circledR}$ ) and blood sampling from the saphenous vein $5 \mathrm{~d}$ later at 16.00 hours. Five dams with glucose $<13 \mathrm{mmol} / \mathrm{l}$ were given a second dosage of STZ, and the increase in glucose was confirmed again $5 \mathrm{~d}$ later. At 1 week after STZ injection (week 2), all the diabetic rats received dosages of insulin (glargine Lantus ${ }^{\circledR}, 0 \cdot 5-10$ unit/d subcutaneously) until postpartum week 4 . Half of the

Table 1. Major composition of the diets $(\mathrm{g} / \mathrm{kg})$

\begin{tabular}{|c|c|c|}
\hline Diet & Control & $\begin{array}{c}\mathrm{AA} \\
(0.5 \mathrm{~g} / 100 \mathrm{~g} \text { fat as } \mathrm{AA})\end{array}$ \\
\hline Maize starch & 629.486 & 629.486 \\
\hline Casein ( $\geq 85 \%)$ & $200 \cdot 0$ & $200 \cdot 0$ \\
\hline Soyabean oil & 70.000 & $69 \cdot 138$ \\
\hline RBD-ARASCO ${ }^{\circledR}(40.6 \%$ AA) & - & 0.862 \\
\hline \multicolumn{3}{|l|}{$\begin{array}{l}\text { Fatty acid composition } \\
\text { (\% of total FA) }\end{array}$} \\
\hline$\Sigma$ SFA & $14 \cdot 70$ & 14.59 \\
\hline$\Sigma$ MUFA & 23.37 & $23 \cdot 17$ \\
\hline $18: 2(n-6)$, LA & $52 \cdot 21$ & 51.93 \\
\hline $18: 3(n-3)$, ALA & $7 \cdot 70$ & $7 \cdot 76$ \\
\hline $20: 4(n-6), \mathrm{AA}$ & 0 & 0.50 \\
\hline$\Sigma$ PUFA & 61.91 & $62 \cdot 24$ \\
\hline
\end{tabular}

AA, arachidonic acid; FA, fatty acid; LA, linoleic acid; ALA, $\alpha$-linolenic acid.

All ingredients were purchased from Harlan Teklad (Madison, WI, USA) except for the AA, which was provided in the form of RBD-ARASCO ${ }^{\circledR}$ (Martek Biosciences Corporation, Columbia, MD, USA). diabetic rats had glucose controlled at $<13 \mathrm{mmol} / \mathrm{l}$ as good control (STZ/GC), and the other half as poor control (STZ/PC) with glucose $13-20 \mathrm{mmol} / \mathrm{l}$. Glucose before insulin management in the STZ/GC group ranged from 14.6 to $30 \cdot 3 \mathrm{mmol} / \mathrm{l}$, while in the STZ/PC group, glucose ranged from 21.8 to $33.3 \mathrm{mmol} / \mathrm{l}$.

The dietary AA intervention started on the same day (week 2) as the insulin treatment. Half of the dams continued on control diet, which was modified from AIN-93G ${ }^{(37)}$ with maize starch as the sole source of carbohydrate. The other half was fed the AA diet, which was the same diet but with $0.5 \mathrm{~g} / 100 \mathrm{~g}$ of fat as AA (RBD-ARASCO: $40.6 \%$ AA; Martek Biosciences Corporation, Columbia, MD, USA).

After the 1-week adaptation period to insulin treatment and diet (week 3), dams were mated and continued on their respective diets and treatments, and the pups were reared by their natural dams until postnatal (PN) week 4. On PN day 3, the pups were weighed and randomised to end points (cull or PN week 4 or 12); then, litters were culled to $\leq 8$ pups, keeping equal numbers of each sex when possible. At PN week 4, half of the pups were euthanised with dams for measurement of fatty acid status at weaning; the other half of the pups were transferred to a regular chow (LabDiet ${ }^{\circledR}$ 5012: $23 \%$ crude protein, $6 \%$ crude fibre and $4.5 \%$ fat) and housed in same-sex groups of up to four per cage until 12 weeks of age (PN week 12) for a long-term assessment. Thus, based on the dams' treatment and diet, the six groups of offspring were as follows: S + C (saline-placebo + control diet); S + A (salineplacebo + AA diet); STZ/GC + C (STZ/GC + control diet); $\mathrm{STZ} / \mathrm{GC}+\mathrm{A}(\mathrm{STZ} / \mathrm{GC}+\mathrm{AA}$ diet $) ; \mathrm{STZ} / \mathrm{PC}+\mathrm{C}(\mathrm{STZ} / \mathrm{PC}+$ control diet); STZ/PC + A (STZ/PC + AA diet).

Throughout the entire study period, dams were housed individually or with pups, and all rats were housed in standard hanging cages with controlled temperature $\left(21-23^{\circ} \mathrm{C}\right)$, humidity $(55 \%)$ and $12 \mathrm{~h}$ light cycle, and fed ad libitum. Dam food intake was monitored daily. The institutional and national guidelines for the care and use of animals were followed, and all the experimental procedures involving animals were approved by the University of Manitoba Protocol Management and Review Committee.

\section{Offspring body composition and bone mass}

Weight was measured weekly for the assessment of growth using a digital scale (Mettler Toledo SB32000, Columbus, $\mathrm{OH}$, USA). At 4,8 and 12 weeks of age, rats were anaesthetised using isoflurane gas ( ${ }^{\mathrm{Pr}}$ AErrane; Baxter Corporation, Mississauga, ON, Canada) for the measurement of bone mass including whole body, lumbar spine, femur and tibia using a small animal program and dual-energy X-ray absorptiometry (DXA, QDR 11.2, 4500A series; Hologic, Bedford, MA, USA). Whole body composition values were obtained for lean mass, fat mass and fat percentage. Whole body length from tip of nose to base of tail was measured in the anaesthetised state using a non-stretchable measuring tape. All scans were performed in singlet with the rat in the posterior position with limbs extended. The DXA measurements have been validated using Hologic hardware and software for rats that are $130 \mathrm{~g}$ and higher in weight for whole body assessments, and in rodents as small as in mice ${ }^{(38)}$ for high-resolution regional scans. 


\section{Offspring oral glucose tolerance test}

Oral glucose tolerance tests (OGTT) were conducted at 8 and 12 weeks of age according to published methods ${ }^{(39)}$ and before the DXA scan. Briefly, after $10 \mathrm{~h}$ without food, blood was collected via saphenous vein for the 0 time point. Immediately following, rats were fed an oral dose of $70 \%$ glucose solution ( $1 \mathrm{~g}$ glucose/kg body weight) using a syringe ${ }^{(39)}$. Blood was collected accordingly at 7.5, 15, 30 and $60 \mathrm{~min}$ after administration of the glucose solution, and blood glucose was determined using a glucometer (One Touch ${ }^{\circledR}$ Ultra ${ }^{\circledR}$ ). The area under the curve (AUC) was calculated from OGTT results using the trapezoidal method ${ }^{(40)}$, which has been validated for glucose tolerance testing ${ }^{(41)}$.

\section{Tissue collection}

At 12 weeks of age, rats were anaesthetised with isoflurane

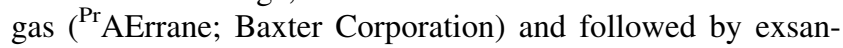
guination via cardiac puncture. Blood was collected, and stored on ice until centrifuged at $1500 \mathrm{rpm}$ for $15 \mathrm{~min}$ at $4^{\circ} \mathrm{C}$. Liver was collected and flash frozen; serum and liver were stored at $-80^{\circ} \mathrm{C}$ until analysis.

\section{Liver fatty acid analysis}

To reflect fatty acid status in response to maternal treatments, liver fatty acids were analysed in dams (postpartum week 4) and offspring (PN weeks 4 and 12). Total lipids from tissues were extracted according to the method from Folch et al. ${ }^{(42)}$. Briefly, after adding an internal standard (C17:0), liver lipid was extracted in chloroform-methanol $(2: 1, \mathrm{v} / \mathrm{v})$ containing butylated hydroxytoluene. Lipid extracts were transmethylated in methanolic $\mathrm{HCl}$ at $80^{\circ} \mathrm{C}$ for $1 \mathrm{~h}$. Fatty acids from twelve to twenty-two carbons were quantified (mg/g tissue) using GC (Varian Star 3400, Mississauga, ON, Canada). Only values for AA and DHA are reported in view of their importance in maternal-fetal transfer. Additionally, DHA has a stronger effect on bone mineral accretion than EPA in growing rats ${ }^{(43)}$.

\section{Statistical analysis}

Data are presented as groups by maternal treatment and diet, offspring sex and age; and they are expressed as means with their standard errors. Main and interaction effects were identified using a mixed (repeated measurements) model procedure of the Statistical Analysis Systems program (version 9.1; SAS Institute, Cary, NC, USA), as it is suitable for the randomised $3 \times 2$ factorial design, and recommended by the University of Manitoba Statistical Advisory Service. Significant differences $(P<0.05)$ among means of groups by maternal treatments or any interaction effects were assessed using Bonferroni's post hoc test.

The correlations between dam mean gestational glucose (mmol/l) and offspring OGTT, DXA scan outcome measurements were detected using Pearson's correlation coefficient $r$ to quantify the strength of the relationship. Correlations between liver AA and DHA (mg/g tissue) from dams and offspring of all ages with offspring OGTT, DXA scan outcome measurements of all ages were examined. Values at 4 weeks of age were averaged per litter per sex to examine longitudinal relationships with same-sex littermates. Values at 8 and 12 weeks of age were available for all offspring, and thus individual values were used in correlation analyses.

\section{Results}

\section{Experimental system}

Induction and control of diabetes were successful as indicated by the mean gestational glucose concentrations at targeted ranges (Table 2). No significant difference was observed among the mean number of pups per litter/group $(P>0.05)$. There were no differences in feed intake between different groups at any given days from mating to delivery (range: $21-36 \mathrm{~g} / \mathrm{d})$ or during lactation $(39-58 \mathrm{~g} / \mathrm{d})$ regardless of treatment and diet.

\section{Offspring body composition}

Total weight gain (from day 3 to 12 weeks) was greater in the STZ/GC offspring compared with STZ/PC offspring regardless of maternal diet $(587.1$ v. $499.5 \%, P=0.009)$, while the saline-placebo offspring were intermediate $(543.7 \%$, $P=0 \cdot 252$ ). Main effects of sex and age were detected in body weight, body length (data not shown), lean mass and fat mass (Table 3 , all $P<0 \cdot 0001$ ). The sex and age effects were manifested as values in male rats being higher than female rats and values at 4 and 8 weeks of age being less than those at 12 weeks of age. There were no main effects (all $P>0.05$ ) of maternal diabetes or AA diet on offspring body weight, body length, lean mass or fat mass.

\section{Offspring bone area, bone mineral content and density}

Maternal diabetes and gestational glucose control had significant effects on bone area, as STZ/PC offspring had smaller bone area than offspring of saline-placebo and STZ/GC at 4 weeks in whole body $(P \leq 0 \cdot 044)$ and at 12 weeks in tibia $(P \leq 0.028)$ (Fig. 1). Femur area of STZ/PC offspring at 8 weeks was less $(P=0.024)$ than that of STZ/GC offspring, while there was no difference between offspring of STZ/PC and saline-placebo. There was no effect of maternal treatment on offspring bone area in lumbar spine $(P>0.05$, data not shown). There was no effect of maternal treatment on offspring bone mineral content (BMC) and bone mineral density (BMD) at any age $(P>0.05)$. Main effects of offspring sex and age were observed for all bone area, BMC and BMD (data not shown) measurements with values increasing over time $(P<0 \cdot 05)$. Values for all treatment groups combined were higher for males than females at 12 weeks for whole body BMC (12.09 (SEM 0.15) v. 8.35 (SEM 0.11) g, $P<0.05$ ) and whole body BMD (0.155 (SEM 0.003) v. 0.149 (SEM $\left.0.002) \mathrm{g} / \mathrm{cm}^{2}, P<0.05\right)$. There was no effect of maternal diet on offspring bone area, BMC and BMD.

\section{Offspring oral glucose tolerance test}

No main effects $(P>0.05)$ of maternal treatment, diet or offspring sex were detected on the OGTT tested at 8 and 12 weeks of age. However, glucose AUC during OGTT 
(AUC glucose ${ }_{0-60}$ ) was greater at 8 weeks than at 12 weeks $(443.7 \quad(\mathrm{SEM} 4.8) \quad v .385 .2 \quad(\mathrm{SEM} \quad 4.5) \mathrm{mmol} / \mathrm{l} \times \mathrm{min}$, $P<0.0001)$ regardless of maternal treatment and diet.

\section{Liver arachidonic acid and DHA}

Dams from STZ/GC plus control diet had significantly lower liver AA than dams from saline-placebo plus AA diet and STZ/PC plus control diet, while those receiving STZ/GC plus AA diet and saline-placebo plus control diet were not different from any group (Table 4). The STZ/GC dams had lower $(P=0.04)$ liver DHA than saline-placebo dams, whereas STZ/PC dams were intermediate.

At 4 weeks, the AA diet offspring had higher $(P<0.004)$ liver AA than control diet offspring. No difference was observed in offspring liver DHA regardless of dams' treatment and diet.

For 12-week-old rats, no difference was observed in offspring liver AA regardless of dams' treatment and diet. The STZ/GC offspring had higher $(P=0.023)$ liver DHA than offspring of STZ/PC and saline-placebo dams. The AA diet offspring had lower $(P=0.030)$ liver DHA than control diet offspring.

At 4 weeks, male rats had higher liver AA and DHA than female rats, while at 12 weeks, the female rats had higher liver DHA than male rats (all $P<0.04)$.

\section{Associations between dam treatment, diet and offspring outcome measurements}

Glucose AUC of OGTT (AUC glucose $_{0-60}$ ) in 8- and 12-week-old offspring did not correlated to any variables that examined including dams' mean gestational glucose, dam (postpartum week 4) and offspring liver AA and DHA (4 and 12 weeks) (data not shown).

Dams' gestational glucose concentration had a significant relationship with bone mass of their offspring at 4 weeks of age. Maternal glucose was negatively correlated with whole body (male: $r-0.41 ; P=0.039$; and female: $r-0.51$; $P=0.013$ ) and lumbar spine (female: $r-0.42 ; P=0.047$ ) bone area (Fig. 2), as well as whole body BMC (male: $r-0.41 ; P=0.039$; and female: $r-0.53 ; P=0.010$ ). A negative correlation appeared at 12 weeks in male rats $(r-0.43$; $P=0.038)$ between maternal glucose and tibia bone area (Fig. 2). In addition, dams' gestational glucose concentration was negatively correlated with offspring whole body fat (male: $r-0.44 ; P=0.030$; and female: $r-0.49 ; P=0.018$ ) and body weight in female rats $(r-0.45 ; P=0.032)$ at 4 weeks, but not thereafter at 8 or 12 weeks.

Maternal liver AA and DHA did not correlated with any measure of bone in 4-week-old offspring. However, in female offspring, maternal DHA was negatively correlated with femur $(r-0.48 ; P=0.030)$ and tibia BMC $(r-0.47$; $P=0.038)$ and tibia BMD $(r-0.50 ; P=0.026)$ at 12 weeks. The only relationships observed between liver fatty acids and bone of the offspring were positive correlations in males between liver AA and lumbar spine BMD $(r$ 0.30; $P=0.05)$ and liver DHA with lumbar spine BMD $(r 0.30 ; P=0.049)$ at 4 weeks of age. In male offspring at 4 weeks, liver AA and DHA were positively correlated $(r \quad 0.86 ; P<0.0001)$. No long-term associations between offspring liver AA were observed with bone area, BMC or BMD (data not shown). 
Table 3. Body composition measured at 4,8 and 12 weeks of age ${ }^{\star}$

(Mean values with their standard errors)

\begin{tabular}{|c|c|c|c|c|c|c|c|c|c|c|c|c|c|c|c|c|c|c|}
\hline & \multirow[b]{2}{*}{ Sex } & \multirow[b]{2}{*}{ Age (weeks) } & \multicolumn{2}{|c|}{$S+C$} & \multicolumn{2}{|c|}{$S+A$} & \multicolumn{2}{|c|}{$S T Z / G C+C$} & \multicolumn{2}{|c|}{$S T Z / G C+A$} & \multicolumn{2}{|c|}{$\mathrm{STZ} / \mathrm{PC}+\mathrm{C}$} & \multicolumn{2}{|c|}{$\mathrm{STZ} / \mathrm{PC}+\mathrm{A}$} & \multicolumn{4}{|c|}{$P$} \\
\hline & & & Mean & SEM & Mean & SEM & Mean & SEM & Mean & SEM & Mean & SEM & Mean & SEM & TRT & Diet & Sex & Age \\
\hline \multirow{4}{*}{$\begin{array}{c}\text { Number of rats at } 4,8 \\
\text { and } 12 \text { weeks }\end{array}$} & $\mathrm{M}$ & 4 & \multicolumn{2}{|c|}{17} & \multicolumn{2}{|c|}{8} & \multicolumn{2}{|c|}{23} & \multicolumn{2}{|c|}{9} & \multicolumn{2}{|c|}{19} & \multicolumn{2}{|c|}{14} & & & & \\
\hline & $\mathrm{F}$ & & \multicolumn{2}{|c|}{15} & \multicolumn{2}{|c|}{12} & 1 & & \multicolumn{2}{|c|}{12} & & & \multicolumn{2}{|c|}{15} & & & & \\
\hline & $\mathrm{M}$ & 8 and 12 & \multicolumn{2}{|c|}{9} & \multicolumn{2}{|c|}{4} & 1 & & \multicolumn{2}{|c|}{5} & & & \multicolumn{2}{|c|}{8} & & & & \\
\hline & $\mathrm{F}$ & & \multicolumn{2}{|c|}{8} & \multicolumn{2}{|c|}{6} & & & \multicolumn{2}{|c|}{6} & & & \multicolumn{2}{|c|}{8} & & & & \\
\hline Body wt (g) & $M$ & 4 & 88.5 & $5 \cdot 0$ & 85.0 & 3.5 & $81 \cdot 2$ & $2 \cdot 3$ & $82 \cdot 7$ & $4 \cdot 1$ & $76 \cdot 1$ & 3.5 & $77 \cdot 7$ & $5 \cdot 7$ & 0.120 & 0.611 & $<0.0001$ & $<0.0001$ \\
\hline & & 8 & 335.6 & $15 \cdot 2$ & $320 \cdot 7$ & 9.5 & 320.9 & $9 \cdot 3$ & 347.3 & $15 \cdot 7$ & 329.4 & $10 \cdot 2$ & 332.1 & $12 \cdot 8$ & & & & \\
\hline & & 12 & $500 \cdot 0$ & $16 \cdot 8$ & 496.4 & $18 \cdot 7$ & 468.9 & 13.0 & 496.9 & 23.4 & 477.8 & $19 \cdot 0$ & 457.0 & $14 \cdot 8$ & & & & \\
\hline & $\mathrm{F}$ & 4 & 81.7 & $4 \cdot 1$ & 78.9 & $3 \cdot 1$ & $73 \cdot 8$ & 2.5 & $78 \cdot 4$ & $3 \cdot 2$ & 69.0 & $5 \cdot 2$ & $70 \cdot 4$ & 3.5 & & & & \\
\hline & & 8 & $204 \cdot 8$ & $6 \cdot 4$ & $212 \cdot 2$ & 3.0 & $212 \cdot 3$ & $5 \cdot 0$ & $207 \cdot 8$ & 7.3 & $187 \cdot 2$ & 9.4 & 205.6 & 8.3 & & & & \\
\hline & & 12 & 272.4 & $8 \cdot 1$ & $272 \cdot 6$ & $8 \cdot 7$ & $277 \cdot 2$ & $6 \cdot 1$ & 281.5 & $17 \cdot 8$ & 260.2 & $12 \cdot 9$ & 255.1 & 8.3 & & & & \\
\hline WB lean (g) & M & 4 & $68 \cdot 3$ & 3.5 & $66 \cdot 3$ & $3 \cdot 2$ & $64 \cdot 3$ & 1.7 & $64 \cdot 6$ & 3.4 & 60.3 & $2 \cdot 5$ & $61 \cdot 1$ & $4 \cdot 8$ & 0.210 & 0.953 & $<0.0001$ & $<0.0001$ \\
\hline & & 8 & 291.0 & $12 \cdot 0$ & $279 \cdot 2$ & 3.5 & 277.6 & $7 \cdot 2$ & 294.7 & $10 \cdot 7$ & 287.0 & 8.0 & 284.8 & $10 \cdot 2$ & & & & \\
\hline & & 12 & 403.0 & 11.7 & $409 \cdot 8$ & $9 \cdot 1$ & 379.8 & $10 \cdot 2$ & 384.0 & $16 \cdot 3$ & 392.6 & $14 \cdot 0$ & 368.8 & $10 \cdot 8$ & & & & \\
\hline & $\mathrm{F}$ & 4 & $62 \cdot 1$ & 2.9 & 61.5 & $2 \cdot 3$ & $57 \cdot 1$ & $2 \cdot 0$ & $62 \cdot 7$ & $2 \cdot 4$ & 53.7 & 3.6 & 54.7 & 3.2 & & & & \\
\hline & & 8 & $183 \cdot 8$ & $5 \cdot 1$ & $187 \cdot 1$ & 4.8 & $192 \cdot 4$ & $3 \cdot 7$ & $189 \cdot 0$ & 5.0 & $182 \cdot 8$ & $7 \cdot 1$ & $179 \cdot 3$ & $4 \cdot 8$ & & & & \\
\hline & & 12 & $220 \cdot 6$ & 3.5 & $229 \cdot 8$ & $5 \cdot 2$ & 227.9 & $5 \cdot 0$ & 215.4 & 6.5 & 220.0 & 9.3 & 211.2 & 6.5 & & & & \\
\hline WB fat $(\mathrm{g})$ & $\mathrm{M}$ & 4 & $18 \cdot 4$ & 1.5 & $16 \cdot 6$ & 0.9 & $15 \cdot 4$ & 0.8 & $16 \cdot 3$ & 0.9 & $14 \cdot 2$ & 1.0 & 14.9 & 0.9 & 0.178 & 0.276 & $<0.0001$ & $<0.0001$ \\
\hline & & 8 & 37.5 & 3.5 & 34.1 & $6 \cdot 3$ & $36 \cdot 2$ & $2 \cdot 8$ & 44.8 & 6.5 & 34.9 & $2 \cdot 6$ & 39.7 & $3 \cdot 2$ & & & & \\
\hline & & 12 & 84.8 & 6.5 & $74 \cdot 3$ & 9.4 & $77 \cdot 2$ & 4.0 & $100 \cdot 0$ & 22.5 & 73.1 & 6.4 & 76.5 & 6.0 & & & & \\
\hline & $\mathrm{F}$ & 4 & $17 \cdot 4$ & 1.5 & $15 \cdot 6$ & 1.0 & 14.8 & 0.7 & $16 \cdot 3$ & 1.1 & 13.7 & 1.6 & 14.1 & 0.5 & & & & \\
\hline & & 8 & $25 \cdot 4$ & $2 \cdot 0$ & 19.9 & 1.7 & 20.4 & $2 \cdot 2$ & $26 \cdot 8$ & 4.0 & 21.0 & $2 \cdot 1$ & 22.6 & 1.4 & & & & \\
\hline & & 12 & 48.5 & $5 \cdot 1$ & 43.8 & 5.4 & $42 \cdot 1$ & 5.5 & 54.7 & 10.7 & 44.1 & 7.2 & 45.5 & 3.4 & & & & \\
\hline
\end{tabular}

S, saline-placebo; C, control diet; A, arachidonic acid diet; STZ, streptozotocin; STZ/GC, STZ-induced diabetes with good control (glucose $<13 \mathrm{mmol} / \mathrm{l}$ ); STZ/PC, STZ-induced diabetes with poor control (glucose 13-20 mmol/); TRT, treatment; M, male; F, female; WB, whole body.

* Sample size was different between 4 weeks and 8,12 weeks as a result of tissue sampling at 4 weeks. 


\section{Discussion}

The most novel observation of the present study is the longterm adverse relationship between maternal hyperglycaemia and skeletal size of adult offspring. Specifically, offspring from poorly controlled diabetic dams (STZ/PC) had significantly smaller bone area of whole body, femur and tibia than offspring from diabetic dams in good control (STZ/ GC). The maternal glucose concentration during pregnancy
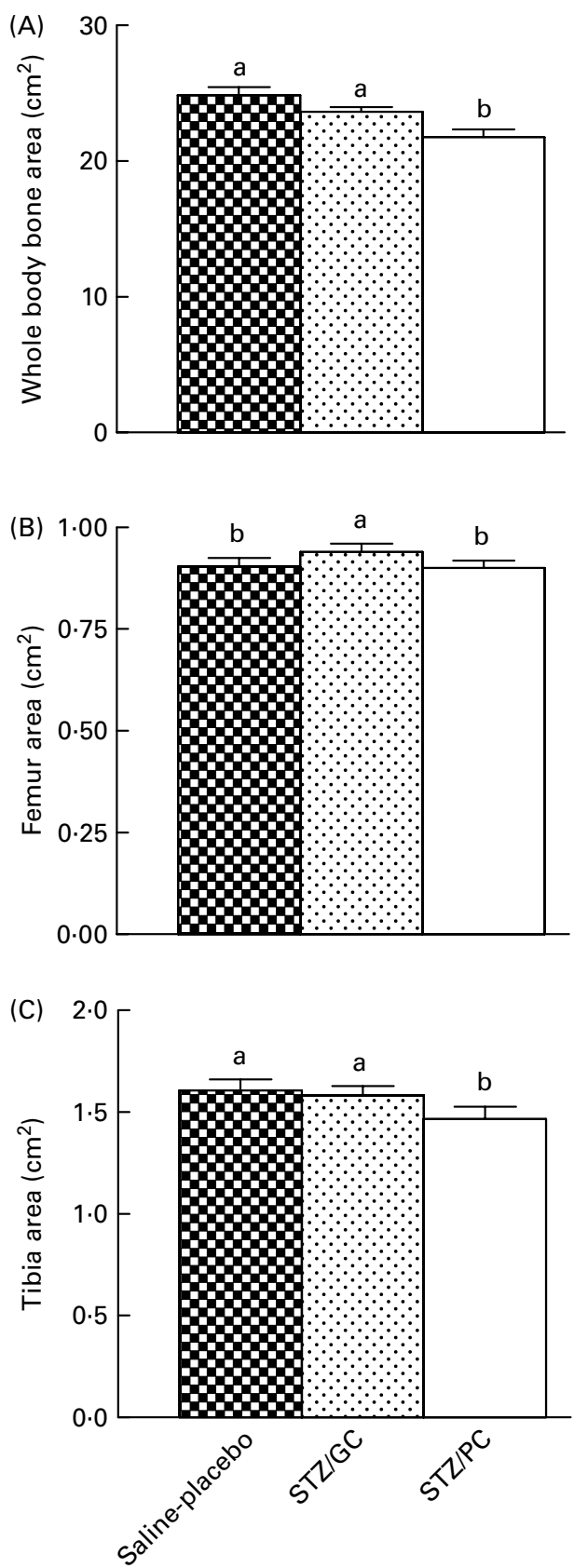

Fig. 1. Bone area $\left(\mathrm{cm}^{2}\right)$ of whole body (4 weeks of age), femur (8 weeks of age) and tibia (12 weeks of age). STZ/GC, STZ-induced diabetes with glucose $<13 \mathrm{mmol} / /$; STZ/PC, STZ-induced diabetes with glucose $13-20 \mathrm{mmol} / \mathrm{l}$. Values are means with their standard errors, which are represented by vertical bars and graph, with unlike letters are significantly different $(P<0.05)$. Sample size is as follows: $(A)$ at 4 weeks, saline-placebo $n 52$, STZ/GC $n$ 57, STZ/PC $n 61 ;((B)$ and (C)) at 8 and 12 weeks, salineplacebo $n 27$, STZ/GC $n 30$, STZ/PC $n 33$.

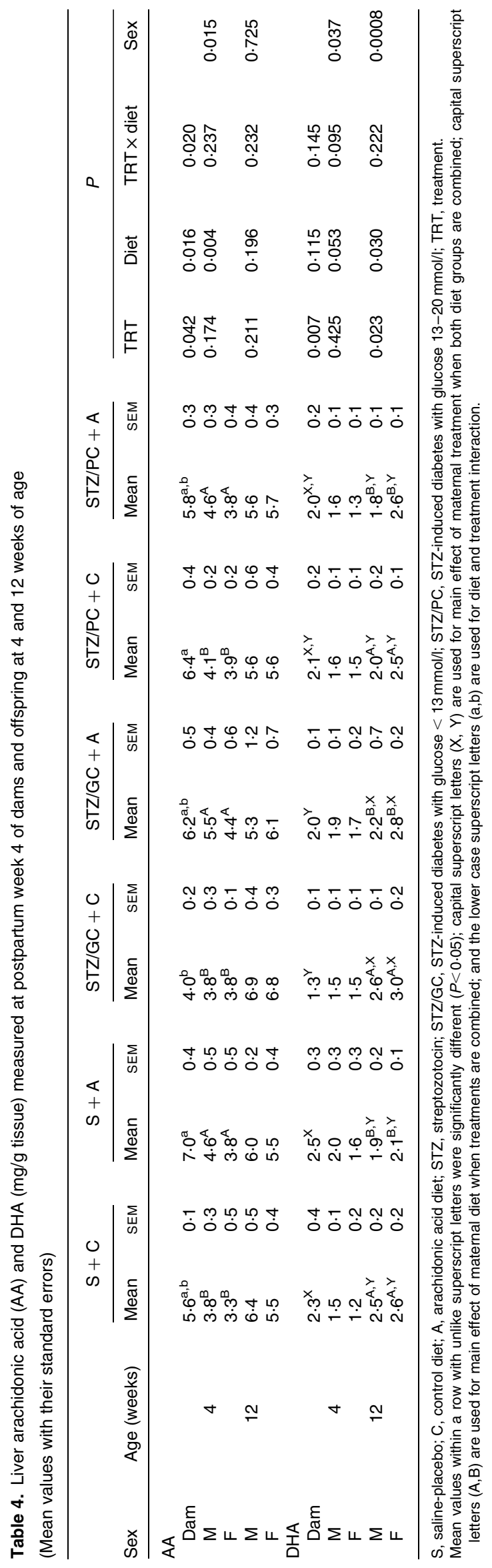



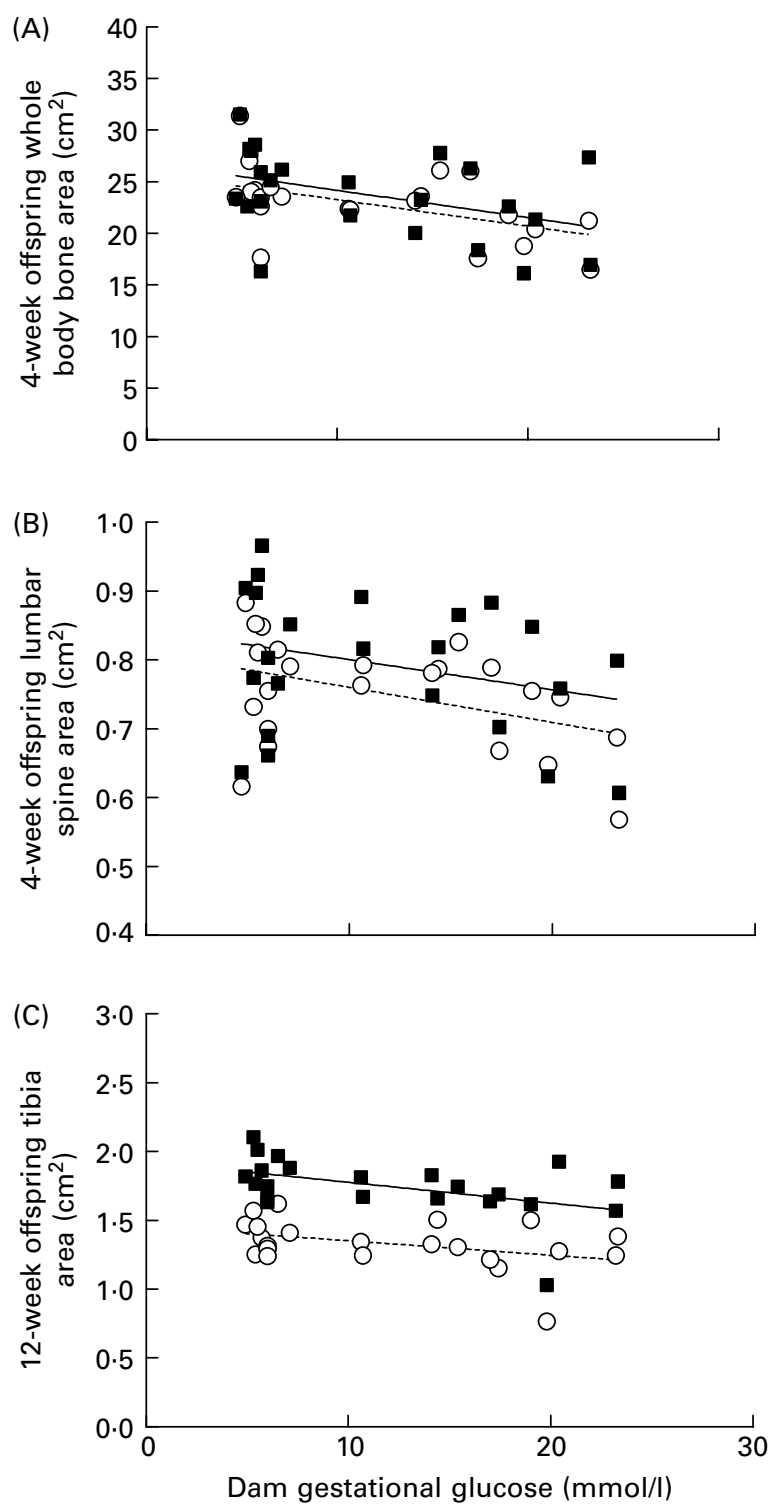

Fig. 2. Correlations between mean gestational glucose concentration $(\mathrm{mmol} / \mathrm{l})$ of dams and offspring bone area $\left(\mathrm{cm}^{2}\right)$ of whole body and lumbar spine at 4 weeks and tibia at 12 weeks of age. Sample size is as follows: at 4 weeks, male $(\mathrm{M}(\square)) n 90$, female $(\mathrm{F}(\bigcirc)) n 80$; at 12 weeks, male $n 48$, female $n$ 42. (A) $\mathrm{M}(r-0.41, P=0.039), \mathrm{F}(r-0.51, P=0.013)$; (B) $\mathrm{M}$ $(r-0.28, P=0.192), \mathrm{F}(r-0.42, P=0.047)$ and $(\mathrm{C}) \mathrm{M}(r-0.43, P=0.038)$, $\mathrm{F}(r-0.39, P=0.071)$.

was inversely related to offspring whole body BMC and bone area at 4 weeks of age and persisted to 12 weeks for tibia area in male rats. This is particularly important in view of the fact that peak bone mass is considered to be well established by 12 weeks of age in rats ${ }^{(44)}$, and therefore suggests that skeletal size is programmed by fetal exposure to maternal hyperglycaemia. Similarly, human infants born to mothers with DM have low $\mathrm{BMC}$ at the distal radius ${ }^{(25)}$, and osteoblastic activity $^{(26)}$ and BMC are inversely related to maternal glucose $^{(25)}$. Furthermore, altered $\mathrm{Ca}$ and $\mathrm{Mg}$ homoeostasis exists in newborn infants of mothers with $\mathrm{DM}^{(24-26)}$ that persists beyond infancy to at least 16 weeks in rats ${ }^{(45)}$ and 10 years in children ${ }^{(46)}$. While it is postulated that this is a compensatory mechanism to support BMD, it appears that cortical density of femur becomes normal while reduced trabecular density persists to 16 weeks in rats ${ }^{(45,47)}$. Correspondingly, at the completion of our 12-week study, BMC and BMD of the STZ offspring were not different from control offspring, although bone architecture was not examined. The present study adds important new information that maternal hyperglycaemia has long-term consequences to bone size of the offspring, implying an altered growth trajectory.

The inverse association between maternal liver DHA with tibia mineral content and density in 12-week-old female offspring is also consistent with the fetal origins of disease hypothesis ${ }^{(48)}$. Even though this hypothesis considers both fetal and neonatal factors, our data suggest that the observations are more closely linked to fetal events since the 12-week measures of BMC and BMD did not relate to liver DHA in neonatal offspring. The mechanism(s) by which DHA exposure in utero might limit BMC and BMD later in life is elusive. Although various eicosanoids act on bone, the PG seem to be the primary mediators of bone cell function ${ }^{(49)}$. $\mathrm{PGE}_{2}$ exhibits biphasic effects on bone, enhancing formation at moderate concentrations but inhibiting formation at both high and low concentrations ${ }^{(50)}$. There is evidence in adult guinea pigs that $\mathrm{PG}$ synthesis is programmed by fetal exposure to long-chain PUFA ${ }^{(51)}$. DHA is known to inhibit osteoclastogenesis ${ }^{(52,53)}$ that is required for modelling of bone during growth ${ }^{(54)}$. Since bone area was not associated with DHA, it is likely that the lower BMC and BMD are linked to altered architecture. Indeed, feeding maternal diets (linseed oil) to elevate fetal exposure to DHA compared to a balance in $n-3: n-6$ fatty acids (soyabean oil) results in lower femur length and lower cortical cross-sectional area and cortical BMC at the mid-diaphysis in 30-week-old female offspring ${ }^{(21,34)}$. Even in human subjects, mothers with higher erythrocytes DHA have neonates with lower spine and femur BMC ${ }^{(55)}$. These three studies imply that higher amounts of DHA in maternal tissue, through an unidentified mechanism in fetal development, programme the fetus for lower BMC and BMD that is apparent as early as infancy in human subjects ${ }^{(55)}$ and continues to adulthood as evidenced in rats ${ }^{(34)}$.

In the present study, maternal diabetes and diet did not affect glucose tolerance in the offspring when measured at 8 and 12 weeks postnatally. To mimic the clinical management of diabetes in pregnant women ${ }^{(56)}$, insulin was used to control glucose, and the offspring were nursed by their natural dams. Thamotharan et al. ${ }^{(57)}$ has reported glucose intolerance, as well as increased food intake and obesity in 60- and 180day-old STZ offspring who were fostered to normal dams, but not in those fostered to diabetic dams. It is possible that our proven method for OGTT $^{(39)}$ was not sufficient ( $1 \mathrm{~g}$ glucose $/ \mathrm{kg}$ body weight) to detect early signs of diabetes in comparison to other studies that used higher dosages and bypassed the gastrointestinal tract $(2 \mathrm{~g} / \mathrm{kg}$ intraperitoneally or intravenously) ${ }^{(58)}$. While it is conceivable that the normal glucose tolerance observed in the present study might have been accompanied by hyperinsulinaemia, a subgroup analysis using 8-week data showed that insulin was 200.0 v. $183.4 \mathrm{pmol} / \mathrm{l}$ at $30 \mathrm{~min}$ into the OGTT in saline-placebo and STZ/GC groups. Thus, hyperinsulinaemia was not a factor in the present study. Future studies should confirm these observations using higher dosages or more sensitive tests such as the euglycaemic-hyperinsulinaemic clamp that 
readily identified glucose intolerance in 12-week-old STZ offspring reared by the biological dams ${ }^{(59)}$.

In addition to the benefits of glucose control in the mother, many studies have postulated that dietary long-chain PUFA will also confer long-term benefits to the offspring. In rats, inclusion of fish oil in the maternal diet during pregnancy offsets the adverse effects of a low-protein diet $(12 \%$, w/w) that otherwise causes insulin resistance in the offspring at 11 months of age ${ }^{(60)}$. Feeding diabetic rat dams with diets containing $35 \%$ of fat as EPA and $6 \%$ of fat as DHA limited the development of insulin resistance in 12-week-old adult offspring compared to the control diet that had a $n-6: n-3$ ratio of $28: 1^{(22)}$. In contrast, in the OGTT, we did not observe any maternal diet effects. In addition to maternal insulin treatment, this could also be ascribed to the standard protein $(20 \%$ in experimental diets and $23 \%$ in regular chow) in all groups and the lower $n-6: n-3$ ratio of $7: 1$. In the present study, the level of dietary supplementation of AA in the dams diet $\left(0.5 \%\right.$ of fat) was based on Otto et al. ${ }^{(61)}$. The present results suggest that such supplementation is safe in regard to longterm growth, body composition and glucose tolerance.

In summary, the present study suggests that maternal hyperglycaemia in pregnancy has a long-lasting adverse effect on skeletal growth in the offspring. Specifically, maternal diabetes was linked to lower tibia area. Regardless of maternal diabetes, higher maternal DHA indirectly related to lower tibia BMC and BMD female offspring at adult ages, suggesting programmed architecture of long bone. Whether the mechanism is ascribed to DHA or eicosanoid metabolism requires further study.

\section{Acknowledgements}

We would like to acknowledge the assistance of Sarah L. Finch, Andrea Maclntosh and Lisa Maximiuk on OGTT, and Gerald Nolette and Denise Borowski for providing diligent animal care and support in University Manitoba Health Sciences Centre animal facility. Each author was involved in the study design, whether in relation to conception of the study or to method development, and was fully involved in study conduct (J. Z.), data analysis (J. Z.) and the final manuscript (all authors). All authors have no conflict of interest. The present study was supported by grants from the Canadian Institute Health Research and the Manitoba Institute of Child Health.

\section{References}

1. Bell R, Bailey K, Cresswell T, et al. (2008) Trends in prevalence and outcomes of pregnancy in women with pre-existing type I and type II diabetes. BJOG 115, 445-452.

2. Freinkel N (1980) Banting Lecture 1980. Of pregnancy and progeny. Diabetes 29, 1023-1035.

3. Metzger BE (2007) Long-term outcomes in mothers diagnosed with gestational diabetes mellitus and their offspring. Clin Obstet Gynecol 50, 972-979.

4. Silverman BL, Metzger BE, Cho NH, et al. (1995) Impaired glucose tolerance in adolescent offspring of diabetic mothers. Relationship to fetal hyperinsulinism. Diabetes Care 18, 611-617.

5. Dabelea D, Mayer-Davis EJ, Lamichhane AP, et al. (2008) Association of intrauterine exposure to maternal diabetes and obesity with type 2 diabetes in youth: the SEARCH CaseControl Study. Diabetes Care 31, 1422-1426.
6. Dixon G, Nolan J, McClenaghan NH, et al. (2004) Arachidonic acid, palmitic acid and glucose are important for the modulation of clonal pancreatic beta-cell insulin secretion, growth and functional integrity. Clin Sci (Lond) 106, 191-199.

7. Konrad RJ, Major CD \& Wolf BA (1994) Diacylglycerol hydrolysis to arachidonic acid is necessary for insulin secretion from isolated pancreatic islets: sequential actions of diacylglycerol and monoacylglycerol lipases. Biochemistry 33, 13284-13294.

8. Borkman M, Storlien LH, Pan DA, et al. (1993) The relation between insulin sensitivity and the fatty-acid composition of skeletal-muscle phospholipids. $N$ Engl J Med 328, 238-244.

9. Sasagawa T, Ishii K, Hasuda K, et al. (2001) The effect of dietary polyunsaturated fatty acid on insulin sensitivity and lipid metabolism in Otsuka Long-Evans Tokushima Fatty (OLETF) rats. Prostaglandins Leukot Essent Fatty Acids 64, 181-187.

10. Wu M, Wang X, Duan Q, et al. (2007) Arachidonic acid can significantly prevent early insulin resistance induced by a high-fat diet. Ann Nutr Metab 51, 270-276.

11. Song MK, Hwang IK, Rosenthal MJ, et al. (2003) Antidiabetic actions of arachidonic acid and zinc in genetically diabetic Goto-Kakizaki rats. Metabolism 52, 7-12.

12. Min Y, Ghebremeskel K, Lowy C, et al. (2004) Adverse effect of obesity on red cell membrane arachidonic and docosahexaenoic acids in gestational diabetes. Diabetologia 47, 75-81.

13. Ortega-Senovilla H, Alvino G, Taricco E, et al. (2009) Gestational diabetes mellitus upsets the proportion of fatty acids in umbilical arterial but not venous plasma. Diabetes Care 32, 120-122.

14. Ghebremeskel K, Thomas B, Lowy C, et al. (2004) Type 1 diabetes compromises plasma arachidonic and docosahexaenoic acids in newborn babies. Lipids 39, 335-342.

15. Min Y, Lowy C, Ghebremeskel K, et al. (2005) Unfavorable effect of type 1 and type 2 diabetes on maternal and fetal essential fatty acid status: a potential marker of fetal insulin resistance. Am J Clin Nutr 82, 1162-1168.

16. Wijendran V, Bendel RB, Couch SC, et al. (2000) Fetal erythrocyte phospholipid polyunsaturated fatty acids are altered in pregnancy complicated with gestational diabetes mellitus. Lipids 35, 927-931.

17. Min Y, Nam JH, Ghebremeskel K, et al. (2006) A distinctive fatty acid profile in circulating lipids of Korean gestational diabetics: a pilot study. Diabetes Res Clin Pract 73, 178-183.

18. Thomas BA, Ghebremeskel K, Lowy C, et al. (2005) Plasma fatty acids of neonates born to mothers with and without gestational diabetes. Prostaglandins Leukot Essent Fatty Acids 72, $335-341$.

19. Ibrahim A, Basak S \& Ehtesham NZ (2009) Impact of maternal dietary fatty acid composition on glucose and lipid metabolism in male rat offspring aged $105 \mathrm{~d}$. Br J Nutr 102, 233-241.

20. Siemelink M, Verhoef A, Dormans JA, et al. (2002) Dietary fatty acid composition during pregnancy and lactation in the rat programs growth and glucose metabolism in the offspring. Diabetologia 45, 1397-1403.

21. Korotkova M, Gabrielsson BG, Holmang A, et al. (2005) Gender-related long-term effects in adult rats by perinatal dietary ratio of $n-6 / n-3$ fatty acids. Am J Physiol Regul Integr Comp Physiol 288, R575-R579.

22. Soulimane-Mokhtari NA, Guermouche B, Yessoufou A, et al. (2005) Modulation of lipid metabolism by $n-3$ polyunsaturated fatty acids in gestational diabetic rats and their macrosomic offspring. Clin Sci (Lond) 109, 287-295.

23. Confavreux CB, Levine RL \& Karsenty G (2009) A paradigm of integrative physiology, the crosstalk between bone and energy metabolisms. Mol Cell Endocrinol 310, 21-29.

24. Tsang RC, Kleinman LI, Sutherland JM, et al. (1972) Hypocalcemia in infants of diabetic mothers. Studies in calcium, phosphorus, and magnesium metabolism and parathormone responsiveness. J Pediatr 80, 384-395. 
25. Mimouni F, Steichen JJ, Tsang RC, et al. (1988) Decreased bone mineral content in infants of diabetic mothers. Am J Perinatol 5, 339-343.

26. Verhaeghe J, Van Herck E \& Bouillon R (1995) Umbilical cord osteocalcin in normal pregnancies and pregnancies complicated by fetal growth retardation or diabetes mellitus. Biol Neonate 68, 377-383.

27. Watkins BA, Li Y \& Seifert MF (2001) Nutraceutical fatty acids as biochemical and molecular modulators of skeletal biology. $J$ Am Coll Nutr 20, 410S-416S, discussion 417S-420S.

28. Weiler HA (2000) Dietary supplementation of arachidonic acid is associated with higher whole body weight and bone mineral density in growing pigs. Pediatr Res 47, 692-697.

29. Watkins BA, Shen CL, McMurtry JP, et al. (1997) Dietary lipids modulate bone prostaglandin $\mathrm{E}_{2}$ production, insulin-like growth factor-I concentration and formation rate in chicks. J Nutr 127, 1084-1091.

30. Alam SQ, Kokkinos PP \& Alam BS (1993) Fatty acid composition and arachidonic acid concentrations in alveolar bone of rats fed diets with different lipids. Calcif Tissue Int 53, $330-332$.

31. Eriksson S, Mellstrom D \& Strandvik B (2009) Fatty acid pattern in serum is associated with bone mineralisation in healthy 8-year-old children. Br J Nutr 102, 407-412.

32. Claassen N, Potgieter HC, Seppa M, et al. (1995) Supplemented gamma-linolenic acid and eicosapentaenoic acid influence bone status in young male rats: effects on free urinary collagen crosslinks, total urinary hydroxyproline, and bone calcium content. Bone 16, 385S-392S.

33. Braddock R, Siman CM, Hamilton K, et al. (2002) Gamma-linoleic acid and ascorbate improves skeletal ossification in offspring of diabetic rats. Pediatr Res 51, 647-652.

34. Korotkova M, Ohlsson C, Hanson LA, et al. (2004) Dietary $n-6: n-3$ fatty acid ratio in the perinatal period affects bone parameters in adult female rats. Br J Nutr 92, 643-648.

35. Korotkova M, Ohlsson C, Gabrielsson B, et al. (2005) Perinatal essential fatty acid deficiency influences body weight and bone parameters in adult male rats. Biochim Biophys Acta 1686, $248-254$.

36. Zhao J, Del Bigio MR \& Weiler HA (2009) Maternal arachidonic acid supplementation improves neurodevelopment of offspring from healthy and diabetic rats. Prostaglandins Leukot Essent Fatty Acids 81, 349-356.

37. Reeves PG (1997) Components of the AIN-93 diets as improvements in the AIN-76A diet. $J$ Nutr 127, 838S-841S.

38. Wlodarski KH \& Dickson GR (2002) Evaluation of locally induced osteoarthritis by the complete and incomplete Freund's adjuvant in mice. The application of DEXA measurements. Folia Biol (Praha) 48, 192-199.

39. Kawa JM, Taylor CG \& Przybylski R (2003) Buckwheat concentrate reduces serum glucose in streptozotocin-diabetic rats. J Agric Food Chem 51, 7287-7291.

40. Purves RD (1992) Optimum numerical integration methods for estimation of area-under-the-curve (AUC) and area-under-themoment-curve (AUMC). J Pharmacokinet Biopharm 20, 211-226.

41. Allison DB, Paultre F, Maggio C, et al. (1995) The use of areas under curves in diabetes research. Diabetes Care 18, 245-250.

42. Folch J, Lees M \& Sloane Stanley GH (1957) A simple method for the isolation and purification of total lipides from animal tissues. J Biol Chem 226, 497-509.

43. Kruger MC \& Schollum LM (2005) Is docosahexaenoic acid more effective than eicosapentaenoic acid for increasing calcium bioavailability? Prostaglandins Leukot Essent Fatty Acids 73, 327-334.

44. Sengupta S, Arshad M, Sharma S, et al. (2005) Attainment of peak bone mass and bone turnover rate in relation to estrous cycle, pregnancy and lactation in colony-bred SpragueDawley rats: suitability for studies on pathophysiology of bone and therapeutic measures for its management. J Steroid Biochem Mol Biol 94, 421-429.

45. Bond H, Hamilton K, Balment RJ, et al. (2005) Diabetes in rat pregnancy alters renal calcium and magnesium reabsorption and bone formation in adult offspring. Diabetologia 48, 1393-1400.

46. Mughal MZ, Eelloo JA, Roberts SA, et al. (2005) Intrauterine programming of urinary calcium and magnesium excretion in children born to mothers with insulin dependent diabetes mellitus. Arch Dis Child Fetal Neonatal Ed 90, F332-F336.

47. Bond H, Sibley CP, Balment RJ, et al. (2005) Increased renal tubular reabsorption of calcium and magnesium by the offspring of diabetic rat pregnancy. Pediatr Res 57, 890-895.

48. Sayer AA \& Cooper C (2005) Fetal programming of body composition and musculoskeletal development. Early Hum Dev 81, 735-744.

49. Watkins BA, Lippman HE, Le Bouteiller L, et al. (2001) Bioactive fatty acids: role in bone biology and bone cell function. Prog Lipid Res 40, 125-148.

50. Raisz LG \& Fall PM (1990) Biphasic effects of prostaglandin $E_{2}$ on bone formation in cultured fetal rat calvariae: interaction with cortisol. Endocrinology 126, 1654-1659.

51. Aprikian O, Reynaud D, Pace-Asciak C, et al. (2007) Neonatal dietary supplementation of arachidonic acid increases prostaglandin levels in adipose tissue but does not promote fat mass development in guinea pigs. Am J Physiol Regul Integr Comp Physiol 293, R2006-R2012.

52. Yuan J, Akiyama M, Nakahama K, et al. (2010) The effects of polyunsaturated fatty acids and their metabolites on osteoclastogenesis in vitro. Prostaglandins Other Lipid Mediat 92, $85-90$.

53. Poulsen RC, Wolber FM, Moughan PJ, et al. (2008) Long chain polyunsaturated fatty acids alter membrane-bound RANK-L expression and osteoprotegerin secretion by MC3T3-E1 osteoblast-like cells. Prostaglandins Other Lipid Mediat 85, 42-48.

54. Narducci P, Bareggi R \& Nicolin V (2009) Receptor activator for nuclear factor kappa B ligand (RANKL) as an osteoimmune key regulator in bone physiology and pathology. Acta Histochem (Epublication ahead of print version 17 November 2009).

55. Weiler H, Fitzpatrick-Wong S, Schellenberg J, et al. (2005) Maternal and cord blood long-chain polyunsaturated fatty acids are predictive of bone mass at birth in healthy term-born infants. Pediatr Res 58, 1254-1258.

56. Walker JD (2008) NICE guidance on diabetes in pregnancy: management of diabetes and its complications from preconception to the postnatal period. NICE clinical guideline 63. London, March 2008. Diabet Med 25, 1025-1027.

57. Thamotharan M, McKnight RA, Thamotharan S, et al. (2003) Aberrant insulin-induced GLUT4 translocation predicts glucose intolerance in the offspring of a diabetic mother. Am J Physiol Endocrinol Metab 284, E901-E914.

58. Han J, Xu J, Long YS, et al. (2007) Rat maternal diabetes impairs pancreatic beta-cell function in the offspring. Am J Physiol Endocrinol Metab 293, E228-E236.

59. Holemans K, Aerts L \& Van Assche FA (1991) Evidence for an insulin resistance in the adult offspring of pregnant streptozotocin-diabetic rats. Diabetologia 34, 81-85.

60. Joshi S, Rao S, Golwilkar A, et al. (2003) Fish oil supplementation of rats during pregnancy reduces adult disease risks in their offspring. J Nutr 133, 3170-3174.

61. Otto SJ, van Houwelingen AC \& Hornstra G (2000) The effect of supplementation with docosahexaenoic and arachidonic acid derived from single cell oils on plasma and erythrocyte fatty acids of pregnant women in the second trimester. Prostaglandins Leukot Essent Fatty Acids 63, 323-328. 